\title{
PLC Implementation as a Flow Computer for Calculation of Saturated Steam Mass Meetings with the Linear Divided Regression Method. (Application: PT. XYZ - Kuala Tanjung)
}

\author{
Dedy AP1, M. Fitra'2, Ahmad AA ${ }^{3}$, Suwarno4 \\ 1,2,3,4 Masters in Electrical Engineering, Universitas Muhammadiyah Sumatera Utara, Indonesia
}

\begin{abstract}
In supporting of production quality improvement, industry must be supported by reliable process system. Reliable measurement system also included in one system which is expected realibility. Specially for saturated steam flow measurement and calculation which is mostly done in the industry in relation to control production cost, should also be able to show accurate results. Now PLC has taken an important role in industry, beside as a control system, PLC also can be used to calculation a formula. This paper aims to use PLC as flow computer to calculate saturatedsteam density by adopting linear regression method which is divided into " $n$ " section to increase the accuration with pressure as a input parameter. The results show a relatively small error value around $0,2 \%$ if we compare with standart linear regression method, where the value around $0,9 \%$.
\end{abstract}

Keyword : Saturated Steam Density, Flow Computer, Linear Regression, PLC.

Corresponding Author:

Dedy AP,

Department of Masters in Electrical Engineering,

Universitas Muhammadiyah Sumatera Utara,

Jalan Kapten Muktar Basri No 3 Medan 20238, Indonesia.

Email: dedi.aputra@yahoo.com

\section{INTRODUCTION}

Currently the industrial world is very strict in terms of quality competition for a product to boost sales. In order to produce good quality products, a production process must be fully supported by a reliable process system. One component of the system is a measurement system.

Steam flow measurement is mostly done in almost every type of industry, because one of the energy sources used is heat energy from steam. Whereas for this flow measuring instrument are very many types, one of which is a type of vortex. In general, the flowmeter vortex can only display the value of the flow size in the form of a volume that is converted from the frequency vortex itself. To get the value of the quantity in the form of mass, a tool is needed to calculate the density value (mass density) which is usually called a flow computer.

To calculate the value of mass flow magnitude can be used the following general equation:

$\mathrm{M}=\mathrm{V} \times \rho$

Where :

$\begin{array}{ll}\mathrm{M} & \text { : Mass flow }(\mathrm{kG} / \text { hour }) \\ \mathrm{V} & : \text { Volume flow }\left(\mathrm{m}^{3} / \text { hour }\right) \\ \rho & \text { : Mass meeting }\left(\mathrm{kG} / \mathrm{m}^{3}\right)\end{array}$

The value of $\rho$ (mass density) itself in a saturated vapor system has a linear relationship that is directly proportional to the value of the vapor pressure. The accuracy of the mass density calculation greatly affects the accuracy of the value of the mass flow, so that the small magnitude of the error value becomes a benchmark of the error value of the steam mass flow. 
In this paper, we will try to offer a method of mass density calculation with a much better level of accuracy through the linear regression method approach adopted into the PLC as an alternative to the flow computer function that is currently widely used in industry.

\section{LITERATURE RIVIEW}

The use of linear regression methods has been used in many writings which aim to determine the relationship between two values or parameters. Linear regression is also widely adopted to formulate several problems into a form of calculation, so that they can be used to forecast or predict a value in the future.

Intan diantari et al (2015) uses linear regression to overcome excess and lack of vehicle stock at Anugrah Utama Motor. Hotanto et al (2015) uses linear regression to forecast fuel sales in the future to avoid losses due to excess stock when there is a change from high to low fuel prices. Multiple linear regression method was used by Jamner R. Lawendatu et al (2014) to analyze the income of nutmeg farmers in Sangihe Islands District - North Sulawesi. As for the prediction of electric power requirements for Lampung Province until 2030 using linear regression has been carried out by M. Syafruddin et al (2014).

In this paper, the linear system method will be implemented as a formula in the PLC program to calculate the mass meeting value in saturated vapor based on the value of the vapor pressure, so that a more accurate value can be obtained.

\section{METHOD}

a. Research Steps.

The steps adopted in this study can be seen in Figure 1 below. Based on the picture, collecting initial data on the relationship between mass meetings and pressure from credible sources is the first thing to do . Then with this data we divide into several segments into " $n$ " to increase its accuracy and calculate the regression value of each segment, where in this case it is determined $n=5$. The formula of each of these ranges is then entered into the PLC program as a formula for calculating steam mass meetings. Then an evaluation of the error between the initial mass meeting data from the reference source and the formula mass meeting data and determine the percentage error.

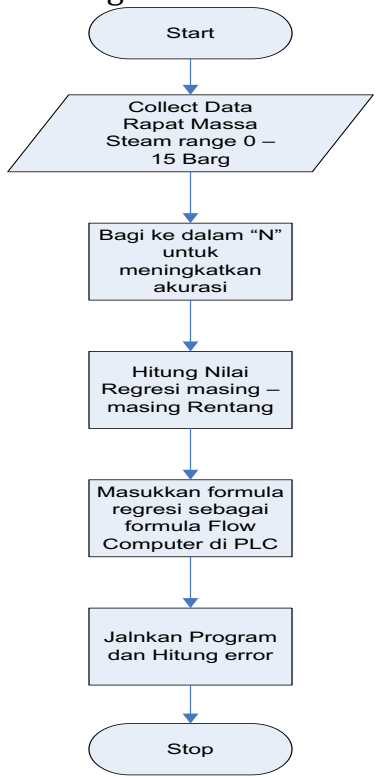

Figure 1. Research Flow Chart

b. Research Equipment .

In this study the equipment that will function as a flow computer is the Nano-Micro Versamax IC200UDR005 PLC with the following specifications.

Brand : General Electric

Series : Versamax Nano / Micro 


$\begin{array}{ll}\text { Power } & : 100 \sim 240 \text { VAC } \\ \text { Input } & : 16 \text { DC Input } \\ \text { Output } & : 11+1 \text { Output }\end{array}$

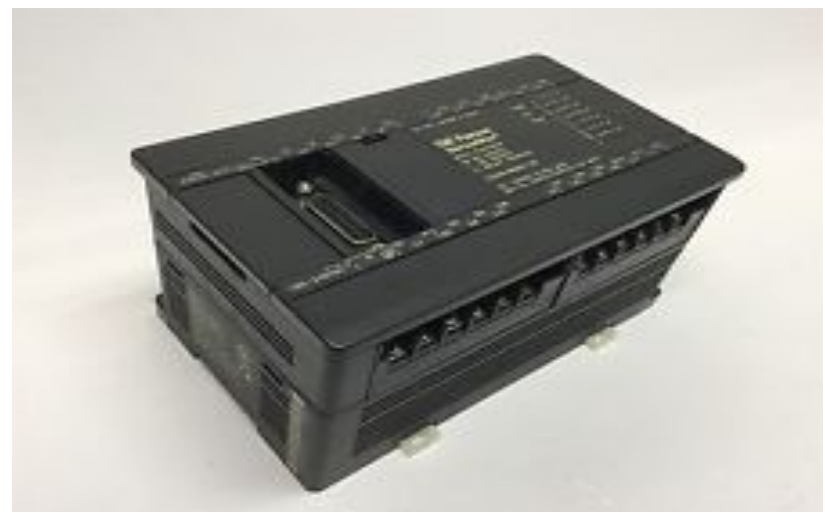

Figure 2. Versamax Nano / Micro PLC.

a. Used Data.

The data used in this study were taken from mass density values based on the saturated vapor pressure of Spirax Sarco database as shown in the following table.

Tabel 1. Vapor pressure-based mass meetings

\begin{tabular}{|c|c|c|c|c|c|c|c|c|c|}
\hline $\begin{array}{c}\text { Pressur } \\
\text { e } \\
\text { (bar } \\
\text { gauge) }\end{array}$ & $\begin{array}{l}\text { Densit } \\
\text { y of } \\
\text { Steam } \\
\left(\mathrm{kg} / \mathrm{m}^{3}\right. \\
\text { ) }\end{array}$ & $\begin{array}{c}\text { Pressur } \\
\text { e } \\
\text { (bar } \\
\text { gauge) }\end{array}$ & $\begin{array}{l}\text { Densit } \\
\text { y of } \\
\text { Steam } \\
\left(\mathrm{kg} / \mathrm{m}^{3}\right. \\
\text { ) }\end{array}$ & $\begin{array}{c}\text { Pressur } \\
\text { e } \\
\text { (bar } \\
\text { gauge) }\end{array}$ & $\begin{array}{l}\text { Densit } \\
\text { y of } \\
\text { Steam } \\
\left(\mathrm{kg} / \mathrm{m}^{3}\right. \\
\text { ) }\end{array}$ & $\begin{array}{c}\text { Pressur } \\
\text { e } \\
\text { (bar } \\
\text { gauge) }\end{array}$ & $\begin{array}{l}\text { Densit } \\
\text { y of } \\
\text { Steam } \\
\left(\mathrm{kg} / \mathrm{m}^{3}\right. \\
\text { ) }\end{array}$ & $\begin{array}{c}\text { Pressur } \\
\text { e } \\
\text { (bar } \\
\text { gauge) }\end{array}$ & $\begin{array}{c}\text { Densit } \\
\text { y of } \\
\text { Steam } \\
\left(\mathrm{kg} / \mathrm{m}^{3}\right. \\
\text { ) }\end{array}$ \\
\hline 0.0 & 0.598 & 3.1 & 2.220 & 6.1 & 3.722 & 9.1 & 5.200 & 12.1 & 6.669 \\
\hline 0.1 & 0.653 & 3.2 & 2.271 & 6.2 & 3.771 & 9.2 & 5.249 & 12.2 & 6.718 \\
\hline 0.2 & 0.707 & 3.3 & 2.321 & 6.3 & 3.821 & 9.3 & 5.298 & 12.3 & 6.767 \\
\hline 0.3 & 0.762 & 3.4 & 2.372 & 6.4 & 3.870 & 9.4 & 5.347 & 12.4 & 6.816 \\
\hline 0.4 & 0.816 & 3.5 & 2.422 & 6.5 & 3.920 & 9.5 & 5.396 & 12.5 & 6.865 \\
\hline 0.5 & 0.870 & 3.6 & 2.473 & 6.6 & 3.969 & 9.6 & 5.445 & 12.6 & 6.914 \\
\hline 0.6 & 0.923 & 3.7 & 2.523 & 6.7 & 4.019 & 9.7 & 5.494 & 12.7 & 6.963 \\
\hline 0.7 & 0.977 & 3.8 & 2.574 & 6.8 & 4.068 & 9.8 & 5.543 & 12.8 & 7.012 \\
\hline 0.8 & 1.030 & 3.9 & 2.624 & 6.9 & 4.117 & 9.9 & 5.592 & 12.9 & 7.060 \\
\hline 0.9 & 1.083 & 4.0 & 2.674 & 7.0 & 4.167 & 10.0 & 5.641 & 13.0 & 7.109 \\
\hline 1.0 & 1.136 & 4.1 & 2.725 & 7.1 & 4.216 & 10.1 & 5.690 & 13.1 & 7.158 \\
\hline 1.1 & 1.189 & 4.2 & 2.775 & 7.2 & 4.265 & 10.2 & 5.739 & 13.2 & 7.207 \\
\hline 1.2 & 1.241 & 4.3 & 2.825 & 7.3 & 4.315 & 10.3 & 5.788 & 13.3 & 7.256 \\
\hline 1.3 & 1.294 & 4.4 & 2.875 & 7.4 & 4.364 & 10.4 & 5.837 & 13.4 & 7.305 \\
\hline 1.4 & 1.346 & 4.5 & 2.925 & 7.5 & 4.413 & 10.5 & 5.886 & 13.5 & 7.354 \\
\hline 1.5 & 1.398 & 4.6 & 2.975 & 7.6 & 4.463 & 10.6 & 5.935 & 13.6 & 7.403 \\
\hline 1.6 & 1.450 & 4.7 & 3.025 & 7.7 & 4.512 & 10.7 & 5.984 & 13.7 & 7.452 \\
\hline 1.7 & 1.502 & 4.8 & 3.075 & 7.8 & 4.561 & 10.8 & 6.033 & 13.8 & 7.501 \\
\hline 1.8 & 1.554 & 4.9 & 3.125 & 7.9 & 4.610 & 10.9 & 6.082 & 13.9 & 7.549 \\
\hline 1.9 & 1.606 & 5.0 & 3.175 & 8.0 & 4.660 & 11.0 & 6.131 & 14.0 & 7.598 \\
\hline
\end{tabular}




\begin{tabular}{llllllllll}
\hline $\mathbf{2 . 0}$ & 1.657 & 5.1 & 3.225 & 8.1 & 4.709 & 11.1 & 6.180 & 14.1 & 7.647 \\
\hline $\mathbf{2 . 1}$ & 1.709 & 5.2 & 3.275 & 8.2 & 4.758 & 11.2 & 6.229 & 14.2 & 7.696 \\
\hline $\mathbf{2 . 2}$ & 1.760 & 5.3 & 3.324 & 8.3 & 4.807 & 11.3 & 6.278 & 14.3 & 7.745 \\
\hline $\mathbf{2 . 3}$ & 1.812 & 5.4 & 3.374 & 8.4 & 4.856 & 11.4 & 6.327 & 14.4 & 7.794 \\
\hline $\mathbf{2 . 4}$ & 1.863 & 5.5 & 3.424 & 8.5 & 4.905 & 11.5 & 6.376 & 14.5 & 7.843 \\
\hline $\mathbf{2 . 5}$ & 1.914 & 5.6 & 3.474 & 8.6 & 4.955 & 11.6 & 6.425 & 14.6 & 7.892 \\
\hline $\mathbf{2 . 6}$ & 1.965 & 5.7 & 3.523 & 8.7 & 5.004 & 11.7 & 6.474 & 14.7 & 7.941 \\
\hline $\mathbf{2 . 7}$ & 2.016 & 5.8 & 3.573 & 8.8 & 5.053 & 11.8 & 6.522 & 14.8 & 7.990 \\
\hline $\mathbf{2 . 8}$ & 2.067 & 5.9 & 3.623 & 8.9 & 5.102 & 11.9 & 6.571 & 14.9 & 8.039 \\
\hline $\mathbf{2 . 9}$ & 2.118 & 6.0 & 3.672 & 9.0 & 5.151 & 12.0 & 6.620 & 15.0 & 8.087 \\
\hline $\mathbf{3 . 0}$ & 2.169 & & & & & & & &
\end{tabular}

\section{RESULTS AND DISCUSSION}

This section will be done formula the data previously obtained by using the linear regression equation to get the steam mass meeting, which had previously been carried division could be presented in the following graph.

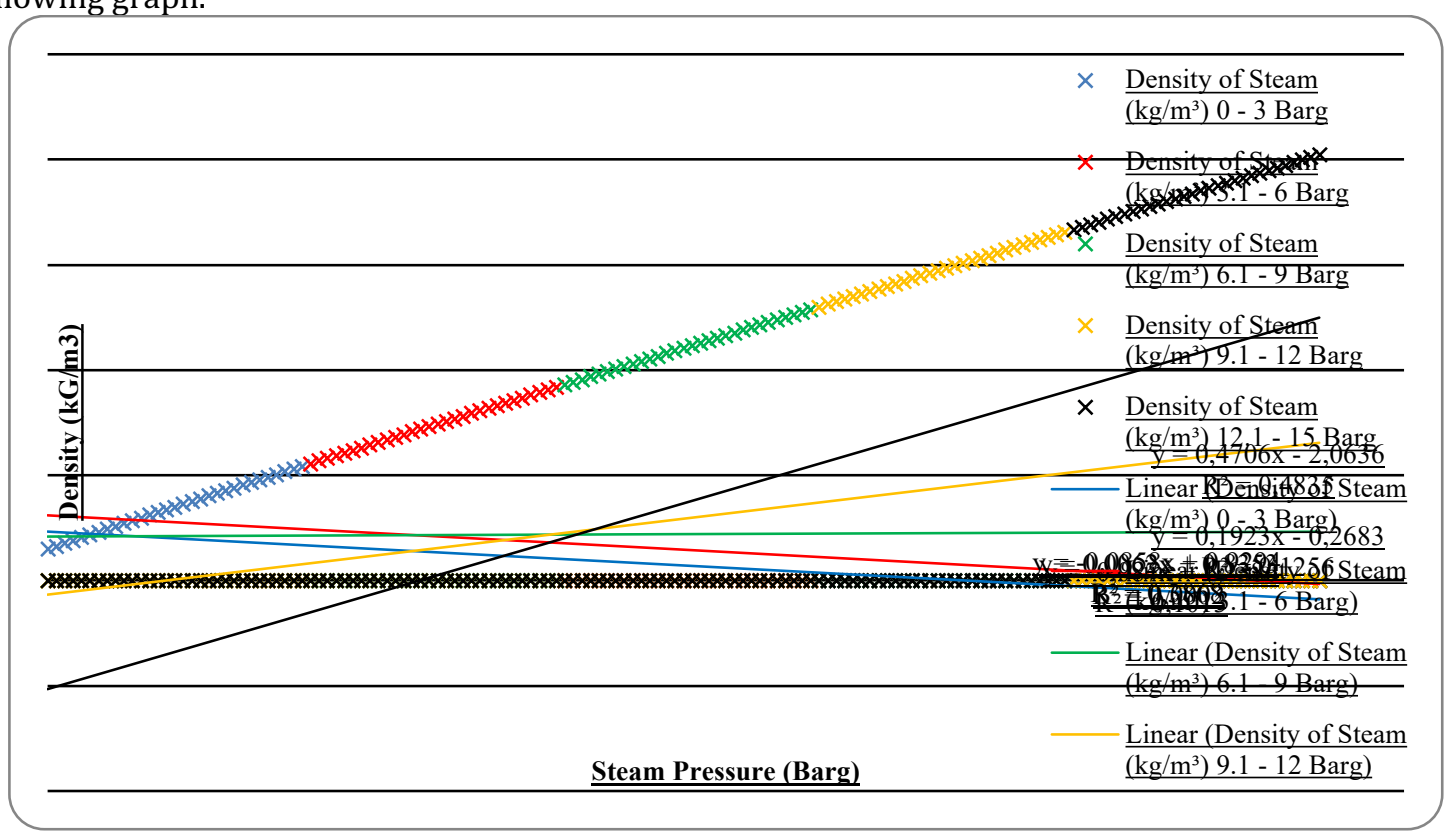

Figure 3. Graph of mass versus vapor pressure.

The graph above shows the relationship between linear mass and pressure meeting values, but in this case the lines in the graph are divided into 5 ranges of values. So there are 5 formulas for each range as follows:

$$
\begin{array}{ll}
\mathrm{y}_{1} & =0,522 \mathrm{x}+0,608 \\
\mathrm{y}_{2} & =0,500 \mathrm{x}+0,670 \\
\mathrm{y}_{3} & =0,492 \mathrm{x}+0,716 \\
\mathrm{y}_{4} & =0,489 \mathrm{x}+0,744 \\
\mathrm{y}_{5} & =0,489 \mathrm{x}+0,752
\end{array}
$$

The formula above is included as a formula for calculating mass meetings into the PLC. After the program starts, the calculation value will appear according to the formula above. Following are the results of the calculation of the program. 
Table 2. Calculation of mass meetings with an interval formula divided by 5

\begin{tabular}{|c|c|c|c|c|c|c|c|c|c|}
\hline $\begin{array}{l}\text { Pressur } \\
\text { e } \\
\text { (bar } \\
\text { gauge) }\end{array}$ & $\begin{array}{l}\text { Densit } \\
\text { y of } \\
\text { Steam } \\
\left(\mathrm{kg} / \mathrm{m}^{3}\right. \\
)^{3}\end{array}$ & $\begin{array}{l}\text { Pressur } \\
\text { e } \\
\text { (bar } \\
\text { gauge) }\end{array}$ & $\begin{array}{l}\text { Densit } \\
\text { y of } \\
\text { Steam } \\
\left(\mathrm{kg} / \mathrm{m}^{3}\right. \\
\text { ) }\end{array}$ & $\begin{array}{l}\text { Pressur } \\
\text { e } \\
\text { (bar } \\
\text { gauge) }\end{array}$ & $\begin{array}{l}\text { Densit } \\
\text { y of } \\
\text { Steam } \\
\left(\mathrm{kg} / \mathrm{m}^{3}\right. \\
\text { ) }\end{array}$ & $\begin{array}{l}\text { Pressur } \\
\text { e } \\
\text { (bar } \\
\text { gauge) }\end{array}$ & $\begin{array}{l}\text { Densit } \\
\text { y of } \\
\text { Steam } \\
\left(\mathrm{kg} / \mathrm{m}^{3}\right. \\
\text { ) }\end{array}$ & $\begin{array}{l}\text { Pressur } \\
\text { e } \\
\text { (bar } \\
\text { gauge) }\end{array}$ & $\begin{array}{l}\text { Densit } \\
\text { y of } \\
\text { Steam } \\
\left(\mathrm{kg} / \mathrm{m}^{3}\right. \\
)^{3}\end{array}$ \\
\hline 0.0 & 0.608 & 3.1 & 2.220 & 6.1 & 3.717 & 9.1 & 5.194 & 12.1 & 6.669 \\
\hline 0.1 & 0.660 & 3.2 & 2.270 & 6.2 & 3.766 & 9.2 & 5.243 & 12.2 & 6.718 \\
\hline 0.2 & 0.712 & 3.3 & 2.320 & 6.3 & 3.816 & 9.3 & 5.292 & 12.3 & 6.767 \\
\hline 0.3 & 0.765 & 3.4 & 2.370 & 6.4 & 3.865 & 9.4 & 5.341 & 12.4 & 6.816 \\
\hline 0.4 & 0.817 & 3.5 & 2.420 & 6.5 & 3.914 & 9.5 & 5.390 & 12.5 & 6.865 \\
\hline 0.5 & 0.869 & 3.6 & 2.470 & 6.6 & 3.963 & 9.6 & 5.438 & 12.6 & 6.913 \\
\hline 0.6 & 0.921 & 3.7 & 2.520 & 6.7 & 4.012 & 9.7 & 5.487 & 12.7 & 6.962 \\
\hline 0.7 & 0.973 & 3.8 & 2.570 & 6.8 & 4.062 & 9.8 & 5.536 & 12.8 & 7.011 \\
\hline 0.8 & 1.026 & 3.9 & 2.620 & 6.9 & 4.111 & 9.9 & 5.585 & 12.9 & 7.060 \\
\hline 0.9 & 1.078 & 4.0 & 2.670 & 7.0 & 4.160 & 10.0 & 5.634 & 13.0 & 7.109 \\
\hline 1.0 & 1.130 & 4.1 & 2.720 & 7.1 & 4.209 & 10.1 & 5.683 & 13.1 & 7.158 \\
\hline 1.1 & 1.182 & 4.2 & 2.770 & 7.2 & 4.258 & 10.2 & 5.732 & 13.2 & 7.207 \\
\hline 1.2 & 1.234 & 4.3 & 2.820 & 7.3 & 4.308 & 10.3 & 5.781 & 13.3 & 7.256 \\
\hline 1.3 & 1.287 & 4.4 & 2.870 & 7.4 & 4.357 & 10.4 & 5.830 & 13.4 & 7.305 \\
\hline 1.4 & 1.339 & 4.5 & 2.920 & 7.5 & 4.406 & 10.5 & 5.879 & 13.5 & 7.354 \\
\hline 1.5 & 1.391 & 4.6 & 2.970 & 7.6 & 4.455 & 10.6 & 5.927 & 13.6 & 7.402 \\
\hline 1.6 & 1.443 & 4.7 & 3.020 & 7.7 & 4.504 & 10.7 & 5.976 & 13.7 & 7.451 \\
\hline 1.7 & 1.495 & 4.8 & 3.070 & 7.8 & 4.554 & 10.8 & 6.025 & 13.8 & 7.500 \\
\hline 1.8 & 1.548 & 4.9 & 3.120 & 7.9 & 4.603 & 10.9 & 6.074 & 13.9 & 7.549 \\
\hline 1.9 & 1.600 & 5.0 & 3.170 & 8.0 & 4.652 & 11.0 & 6.123 & 14.0 & 7.598 \\
\hline 2.0 & 1.652 & 5.1 & 3.220 & 8.1 & 4.701 & 11.1 & 6.172 & 14.1 & 7.647 \\
\hline 2.1 & 1.704 & 5.2 & 3.270 & 8.2 & 4.750 & 11.2 & 6.221 & 14.2 & 7.696 \\
\hline 2.2 & 1.756 & 5.3 & 3.320 & 8.3 & 4.800 & 11.3 & 6.270 & 14.3 & 7.745 \\
\hline 2.3 & 1.809 & 5.4 & 3.370 & 8.4 & 4.849 & 11.4 & 6.319 & 14.4 & 7.794 \\
\hline 2.4 & 1.861 & 5.5 & 3.420 & 8.5 & 4.898 & 11.5 & 6.368 & 14.5 & 7.843 \\
\hline 2.5 & 1.913 & 5.6 & 3.470 & 8.6 & 4.947 & 11.6 & 6.416 & 14.6 & 7.891 \\
\hline 2.6 & 1.965 & 5.7 & 3.520 & 8.7 & 4.996 & 11.7 & 6.465 & 14.7 & 7.940 \\
\hline 2.7 & 2.017 & 5.8 & 3.570 & 8.8 & 5.046 & 11.8 & 6.514 & 14.8 & 7.989 \\
\hline 2.8 & 2.070 & 5.9 & 3.620 & 8.9 & 5.095 & 11.9 & 6.563 & 14.9 & 8.038 \\
\hline 2.9 & 2.122 & 6.0 & 3.670 & 9.0 & 5.144 & 12.0 & 6.612 & 15.0 & 8.087 \\
\hline 3.0 & 2.174 & & & & & & & & \\
\hline
\end{tabular}

To see accuracy needs to be calculated relative error values as shown in the following table, where the average percentage error value of the overall value is $0.2 \%$.

Table 3. Calculation of mass meeting errors with a formula of 5 values. 


\begin{tabular}{|c|c|c|c|c|c|c|c|c|c|}
\hline $\begin{array}{l}\text { Pressur } \\
\text { e } \\
\text { (bar } \\
\text { gauge) }\end{array}$ & $\begin{array}{l}\text { Densit } \\
\text { y of } \\
\text { Steam } \\
\left(\mathrm{kg} / \mathrm{m}^{3}\right. \\
\text { ) }\end{array}$ & $\begin{array}{l}\text { Pressur } \\
\text { e } \\
\text { (bar } \\
\text { gauge) }\end{array}$ & $\begin{array}{l}\text { Densit } \\
\text { y of } \\
\text { Steam } \\
\left(\mathrm{kg} / \mathrm{m}^{3}\right. \\
\text { ) }\end{array}$ & $\begin{array}{l}\text { Pressur } \\
\text { e } \\
\text { (bar } \\
\text { gauge) }\end{array}$ & $\begin{array}{l}\text { Densit } \\
\text { y of } \\
\text { Steam } \\
\left(\mathrm{kg} / \mathrm{m}^{3}\right. \\
\text { ) }\end{array}$ & $\begin{array}{l}\text { Pressur } \\
\mathrm{e} \\
\text { (bar } \\
\text { gauge) }\end{array}$ & $\begin{array}{l}\text { Densit } \\
\text { y of } \\
\text { Steam } \\
\left(\mathrm{kg} / \mathrm{m}^{3}\right. \\
\text { ) }\end{array}$ & $\begin{array}{l}\text { Pressur } \\
\text { e } \\
\text { (bar } \\
\text { gauge) }\end{array}$ & $\begin{array}{l}\text { Densit } \\
\text { y of } \\
\text { Steam } \\
\left(\mathrm{kg} / \mathrm{m}^{3}\right. \\
{ }^{3}\end{array}$ \\
\hline 0.0 & $1.8 \%$ & 3.1 & $0.0 \%$ & 6.1 & $0.1 \%$ & 9.1 & $0.1 \%$ & 12.1 & $0.0 \%$ \\
\hline 0.1 & $1.2 \%$ & 3.2 & $0.0 \%$ & 6.2 & $0.1 \%$ & 9.2 & $0.1 \%$ & 12.2 & $0.0 \%$ \\
\hline 0.2 & $0.7 \%$ & 3.3 & $0.1 \%$ & 6.3 & $0.1 \%$ & 9.3 & $0.1 \%$ & 12.3 & $0.0 \%$ \\
\hline 0.3 & $0.4 \%$ & 3.4 & $0.1 \%$ & 6.4 & $0.1 \%$ & 9.4 & $0.1 \%$ & 12.4 & $0.0 \%$ \\
\hline 0.4 & $0.1 \%$ & 3.5 & $0.1 \%$ & 6.5 & $0.1 \%$ & 9.5 & $0.1 \%$ & 12.5 & $0.0 \%$ \\
\hline 0.5 & $0.1 \%$ & 3.6 & $0.1 \%$ & 6.6 & $0.2 \%$ & 9.6 & $0.1 \%$ & 12.6 & $0.0 \%$ \\
\hline 0.6 & $0.2 \%$ & 3.7 & $0.1 \%$ & 6.7 & $0.2 \%$ & 9.7 & $0.1 \%$ & 12.7 & $0.0 \%$ \\
\hline 0.7 & $0.3 \%$ & 3.8 & $0.1 \%$ & 6.8 & $0.2 \%$ & 9.8 & $0.1 \%$ & 12.8 & $0.0 \%$ \\
\hline 0.8 & $0.4 \%$ & 3.9 & $0.2 \%$ & 6.9 & $0.2 \%$ & 9.9 & $0.1 \%$ & 12.9 & $0.0 \%$ \\
\hline 0.9 & $0.5 \%$ & 4.0 & $0.2 \%$ & 7.0 & $0.2 \%$ & 10.0 & $0.1 \%$ & 13.0 & $0.0 \%$ \\
\hline 1.0 & $0.5 \%$ & 4.1 & $0.2 \%$ & 7.1 & $0.2 \%$ & 10.1 & $0.1 \%$ & 13.1 & $0.0 \%$ \\
\hline 1.1 & $0.5 \%$ & 4.2 & $0.2 \%$ & 7.2 & $0.2 \%$ & 10.2 & $0.1 \%$ & 13.2 & $0.0 \%$ \\
\hline 1.2 & $0.5 \%$ & 4.3 & $0.2 \%$ & 7.3 & $0.2 \%$ & 10.3 & $0.1 \%$ & 13.3 & $0.0 \%$ \\
\hline 1.3 & $0.5 \%$ & 4.4 & $0.2 \%$ & 7.4 & $0.2 \%$ & 10.4 & $0.1 \%$ & 13.4 & $0.0 \%$ \\
\hline 1.4 & $0.5 \%$ & 4.5 & $0.2 \%$ & 7.5 & $0.2 \%$ & 10.5 & $0.1 \%$ & 13.5 & $0.0 \%$ \\
\hline 1.5 & $0.5 \%$ & 4.6 & $0.2 \%$ & 7.6 & $0.2 \%$ & 10.6 & $0.1 \%$ & 13.6 & $0.0 \%$ \\
\hline 1.6 & $0.5 \%$ & 4.7 & $0.2 \%$ & 7.7 & $0.2 \%$ & 10.7 & $0.1 \%$ & 13.7 & $0.0 \%$ \\
\hline 1.7 & $0.4 \%$ & 4.8 & $0.2 \%$ & 7.8 & $0.2 \%$ & 10.8 & $0.1 \%$ & 13.8 & $0.0 \%$ \\
\hline 1.8 & $0.4 \%$ & 4.9 & $0.2 \%$ & 7.9 & $0.2 \%$ & 10.9 & $0.1 \%$ & 13.9 & $0.0 \%$ \\
\hline 1.9 & $0.4 \%$ & 5.0 & $0.2 \%$ & 8.0 & $0.2 \%$ & 11.0 & $0.1 \%$ & 14.0 & $0.0 \%$ \\
\hline 2.0 & $0.3 \%$ & 5.1 & $0.1 \%$ & 8.1 & $0.2 \%$ & 11.1 & $0.1 \%$ & 14.1 & $0.0 \%$ \\
\hline 2.1 & $0.3 \%$ & 5.2 & $0.1 \%$ & 8.2 & $0.2 \%$ & 11.2 & $0.1 \%$ & 14.2 & $0.0 \%$ \\
\hline 2.2 & $0.2 \%$ & 5.3 & $0.1 \%$ & 8.3 & $0.2 \%$ & 11.3 & $0.1 \%$ & 14.3 & $0.0 \%$ \\
\hline 2.3 & $0.2 \%$ & 5.4 & $0.1 \%$ & 8.4 & $0.2 \%$ & 11.4 & $0.1 \%$ & 14.4 & $0.0 \%$ \\
\hline 2.4 & $0.1 \%$ & 5.5 & $0.1 \%$ & 8.5 & $0.2 \%$ & 11.5 & $0.1 \%$ & 14.5 & $0.0 \%$ \\
\hline 2.5 & $0.1 \%$ & 5.6 & $0.1 \%$ & 8.6 & $0.1 \%$ & 11.6 & $0.1 \%$ & 14.6 & $0.0 \%$ \\
\hline 2.6 & $0.0 \%$ & 5.7 & $0.1 \%$ & 8.7 & $0.1 \%$ & 11.7 & $0.1 \%$ & 14.7 & $0.0 \%$ \\
\hline 2.7 & $0.1 \%$ & 5.8 & $0.1 \%$ & 8.8 & $0.1 \%$ & 11.8 & $0.1 \%$ & 14.8 & $0.0 \%$ \\
\hline 2.8 & $0.1 \%$ & 5.9 & $0.1 \%$ & 8.9 & $0.1 \%$ & 11.9 & $0.1 \%$ & 14.9 & $0.0 \%$ \\
\hline 2.9 & $0.2 \%$ & 6.0 & $0.1 \%$ & 9.0 & $0.1 \%$ & 12.0 & $0.1 \%$ & 15.0 & $0.0 \%$ \\
\hline 3.0 & $0.2 \%$ & & & & & & & & \\
\hline
\end{tabular}




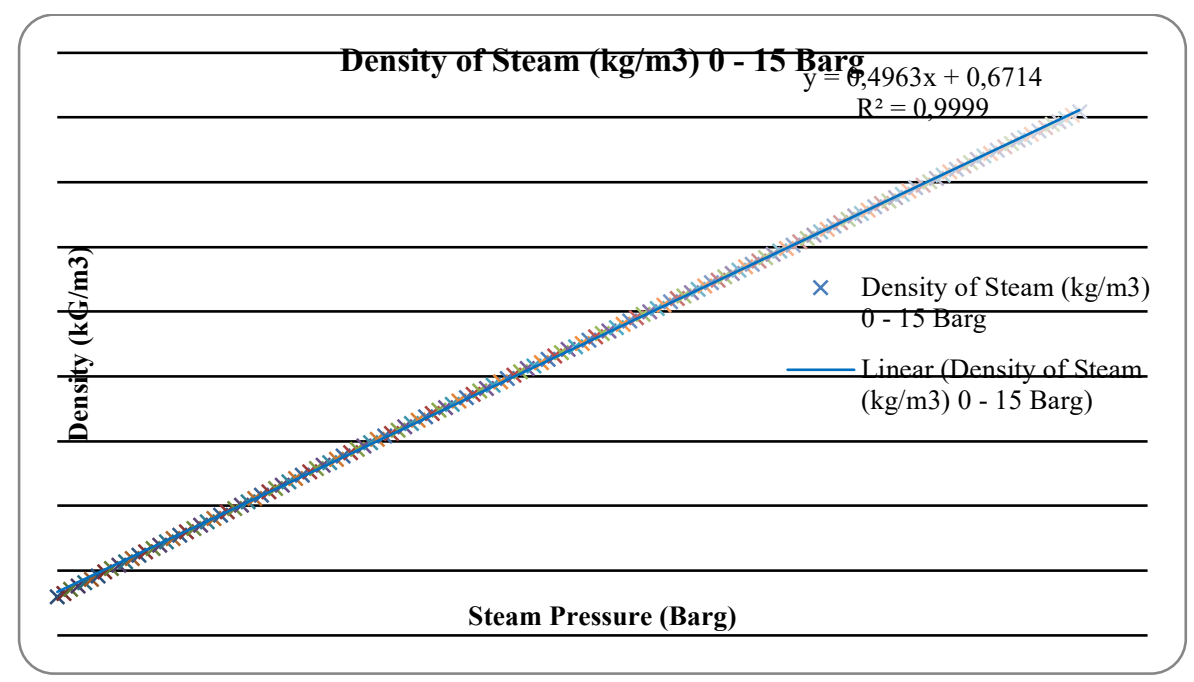

Figure 4. Graph of mass density vs. vapor pressure regression without being divided into $\mathrm{n}$. of values :

From the graph above, the regression formula can be determined without the following range

$$
\mathrm{y} \quad=0,496 \mathrm{x}+0,671
$$
follows:

By formulating the above equation into the PLC program, the mass meeting values are as

Table 4. Calculations of mass meetings without ranges are divided into $\mathrm{n}$

\begin{tabular}{|c|c|c|c|c|c|c|c|c|c|}
\hline $\begin{array}{l}\text { Pressur } \\
\text { e } \\
\text { (bar } \\
\text { gauge) }\end{array}$ & $\begin{array}{l}\text { Densit } \\
\text { y of } \\
\text { Steam } \\
\left(\mathrm{kg} / \mathrm{m}^{3}\right. \\
\text { ) }\end{array}$ & $\begin{array}{l}\text { Pressur } \\
\text { e } \\
\text { (bar } \\
\text { gauge) }\end{array}$ & $\begin{array}{l}\text { Densit } \\
\text { y of } \\
\text { Steam } \\
\left(\mathrm{kg} / \mathrm{m}^{3}\right. \\
\text { ) }\end{array}$ & $\begin{array}{l}\text { Pressur } \\
\text { e } \\
\text { (bar } \\
\text { gauge) }\end{array}$ & $\begin{array}{l}\text { Densit } \\
\text { y of } \\
\text { Steam } \\
\left(\mathrm{kg} / \mathrm{m}^{3}\right. \\
\text { ) }\end{array}$ & $\begin{array}{l}\text { Pressur } \\
\text { e } \\
\text { (bar } \\
\text { gauge) }\end{array}$ & $\begin{array}{l}\text { Densit } \\
\text { y of } \\
\text { Steam } \\
\left(\mathrm{kg} / \mathrm{m}^{3}\right. \\
\text { ) }\end{array}$ & $\begin{array}{l}\text { Pressur } \\
\text { e } \\
\text { (bar } \\
\text { gauge) }\end{array}$ & $\begin{array}{l}\text { Densit } \\
\text { y of } \\
\text { Steam } \\
\left(\mathrm{kg} / \mathrm{m}^{3}\right. \\
{ }^{3}\end{array}$ \\
\hline 0.0 & 0.671 & 3.1 & 2.209 & 6.1 & 3.697 & 9.1 & 5.185 & 12.1 & 6.673 \\
\hline 0.1 & 0.721 & 3.2 & 2.258 & 6.2 & 3.746 & 9.2 & 5.234 & 12.2 & 6.722 \\
\hline 0.2 & 0.770 & 3.3 & 2.308 & 6.3 & 3.796 & 9.3 & 5.284 & 12.3 & 6.772 \\
\hline 0.3 & 0.820 & 3.4 & 2.357 & 6.4 & 3.845 & 9.4 & 5.333 & 12.4 & 6.821 \\
\hline 0.4 & 0.869 & 3.5 & 2.407 & 6.5 & 3.895 & 9.5 & 5.383 & 12.5 & 6.871 \\
\hline 0.5 & 0.919 & 3.6 & 2.457 & 6.6 & 3.945 & 9.6 & 5.433 & 12.6 & 6.921 \\
\hline 0.6 & 0.969 & 3.7 & 2.506 & 6.7 & 3.994 & 9.7 & 5.482 & 12.7 & 6.970 \\
\hline 0.7 & 1.018 & 3.8 & 2.556 & 6.8 & 4.044 & 9.8 & 5.532 & 12.8 & 7.020 \\
\hline 0.8 & 1.068 & 3.9 & 2.605 & 6.9 & 4.093 & 9.9 & 5.581 & 12.9 & 7.069 \\
\hline 0.9 & 1.117 & 4.0 & 2.655 & 7.0 & 4.143 & 10.0 & 5.631 & 13.0 & 7.119 \\
\hline 1.0 & 1.167 & 4.1 & 2.705 & 7.1 & 4.193 & 10.1 & 5.681 & 13.1 & 7.169 \\
\hline 1.1 & 1.217 & 4.2 & 2.754 & 7.2 & 4.242 & 10.2 & 5.730 & 13.2 & 7.218 \\
\hline 1.2 & 1.266 & 4.3 & 2.804 & 7.3 & 4.292 & 10.3 & 5.780 & 13.3 & 7.268 \\
\hline 1.3 & 1.316 & 4.4 & 2.853 & 7.4 & 4.341 & 10.4 & 5.829 & 13.4 & 7.317 \\
\hline 1.4 & 1.365 & 4.5 & 2.903 & 7.5 & 4.391 & 10.5 & 5.879 & 13.5 & 7.367 \\
\hline 1.5 & 1.415 & 4.6 & 2.953 & 7.6 & 4.441 & 10.6 & 5.929 & 13.6 & 7.417 \\
\hline 1.6 & 1.465 & 4.7 & 3.002 & 7.7 & 4.490 & 10.7 & 5.978 & 13.7 & 7.466 \\
\hline 1.7 & 1.514 & 4.8 & 3.052 & 7.8 & 4.540 & 10.8 & 6.028 & 13.8 & 7.516 \\
\hline
\end{tabular}




\begin{tabular}{llllllllll}
\hline $\mathbf{1 . 8}$ & 1.564 & 4.9 & 3.101 & 7.9 & 4.589 & 10.9 & 6.077 & 13.9 & 7.565 \\
\hline $\mathbf{1 . 9}$ & 1.613 & 5.0 & 3.151 & 8.0 & 4.639 & 11.0 & 6.127 & 14.0 & 7.615 \\
\hline $\mathbf{2 . 0}$ & 1.663 & 5.1 & 3.201 & 8.1 & 4.689 & 11.1 & 6.177 & 14.1 & 7.665 \\
\hline $\mathbf{2 . 1}$ & 1.713 & 5.2 & 3.250 & 8.2 & 4.738 & 11.2 & 6.226 & 14.2 & 7.714 \\
\hline $\mathbf{2 . 2}$ & 1.762 & 5.3 & 3.300 & 8.3 & 4.788 & 11.3 & 6.276 & 14.3 & 7.764 \\
\hline $\mathbf{2 . 3}$ & 1.812 & 5.4 & 3.349 & 8.4 & 4.837 & 11.4 & 6.325 & 14.4 & 7.813 \\
\hline $\mathbf{2 . 4}$ & 1.861 & 5.5 & 3.399 & 8.5 & 4.887 & 11.5 & 6.375 & 14.5 & 7.863 \\
\hline $\mathbf{2 . 5}$ & 1.911 & 5.6 & 3.449 & 8.6 & 4.937 & 11.6 & 6.425 & 14.6 & 7.913 \\
\hline $\mathbf{2 . 6}$ & 1.961 & 5.7 & 3.498 & 8.7 & 4.986 & 11.7 & 6.474 & 14.7 & 7.962 \\
\hline $\mathbf{2 . 7}$ & 2.010 & 5.8 & 3.548 & 8.8 & 5.036 & 11.8 & 6.524 & 14.8 & 8.012 \\
\hline $\mathbf{2 . 8}$ & 2.060 & 5.9 & 3.597 & 8.9 & 5.085 & 11.9 & 6.573 & 14.9 & 8.061 \\
\hline $\mathbf{2 . 9}$ & 2.109 & 6.0 & 3.647 & 9.0 & 5.135 & 12.0 & 6.623 & 15.0 & 8.111 \\
\hline $\mathbf{3 . 0}$ & 2.159 & & & & & & & &
\end{tabular}

With a percentage error of $0.9 \%$ as shown in the following table :

Table 5. Calculation of mass density errors without value ranges

\begin{tabular}{|c|c|c|c|c|c|c|c|c|c|}
\hline $\begin{array}{l}\text { Pressur } \\
\text { e } \\
\text { (bar } \\
\text { gauge) }\end{array}$ & $\begin{array}{l}\text { Densit } \\
\text { y of } \\
\text { Steam } \\
\text { (kg/m } / \mathrm{m}^{3} \\
\text { ) }\end{array}$ & $\begin{array}{l}\text { Pressur } \\
\text { e } \\
\text { (bar } \\
\text { gauge) }\end{array}$ & $\begin{array}{l}\text { Densit } \\
\text { y of } \\
\text { Steam } \\
\left(\mathrm{kg} / \mathrm{m}^{3}\right. \\
\text { ) }\end{array}$ & $\begin{array}{l}\text { Pressur } \\
\text { e } \\
\text { (bar } \\
\text { gauge) }\end{array}$ & $\begin{array}{l}\text { Densit } \\
\text { y of } \\
\text { Steam } \\
\left(\mathrm{kg} / \mathrm{m}^{3}\right. \\
)\end{array}$ & $\begin{array}{l}\text { Pressur } \\
\mathrm{e} \\
\text { (bar } \\
\text { gauge) }\end{array}$ & $\begin{array}{l}\text { Densit } \\
\text { y of } \\
\text { Steam } \\
\left(\mathrm{kg} / \mathrm{m}^{3}\right. \\
\text { ) }\end{array}$ & $\begin{array}{l}\text { Pressur } \\
\text { e } \\
\text { (bar } \\
\text { gauge) }\end{array}$ & $\begin{array}{l}\text { Densit } \\
\text { y of } \\
\text { Steam } \\
\left(\mathrm{kg} / \mathrm{m}^{3}\right. \\
\text { ) }\end{array}$ \\
\hline 0.0 & $12.3 \%$ & 3.1 & $0.5 \%$ & 6.1 & $0.7 \%$ & 9.1 & $0.3 \%$ & 12.1 & $0.1 \%$ \\
\hline 0.1 & $10.4 \%$ & 3.2 & $0.5 \%$ & 6.2 & $0.7 \%$ & 9.2 & $0.3 \%$ & 12.2 & $0.1 \%$ \\
\hline 0.2 & $8.9 \%$ & 3.3 & $0.6 \%$ & 6.3 & $0.7 \%$ & 9.3 & $0.3 \%$ & 12.3 & $0.1 \%$ \\
\hline 0.3 & $7.6 \%$ & 3.4 & $0.6 \%$ & 6.4 & $0.6 \%$ & 9.4 & $0.3 \%$ & 12.4 & $0.1 \%$ \\
\hline 0.4 & $6.6 \%$ & 3.5 & $0.6 \%$ & 6.5 & $0.6 \%$ & 9.5 & $0.2 \%$ & 12.5 & $0.1 \%$ \\
\hline 0.5 & $5.7 \%$ & 3.6 & $0.7 \%$ & 6.6 & $0.6 \%$ & 9.6 & $0.2 \%$ & 12.6 & $0.1 \%$ \\
\hline 0.6 & $4.9 \%$ & 3.7 & $0.7 \%$ & 6.7 & $0.6 \%$ & 9.7 & $0.2 \%$ & 12.7 & $0.1 \%$ \\
\hline 0.7 & $4.3 \%$ & 3.8 & $0.7 \%$ & 6.8 & $0.6 \%$ & 9.8 & $0.2 \%$ & 12.8 & $0.1 \%$ \\
\hline 0.8 & $3.7 \%$ & 3.9 & $0.7 \%$ & 6.9 & $0.6 \%$ & 9.9 & $0.2 \%$ & 12.9 & $0.1 \%$ \\
\hline 0.9 & $3.2 \%$ & 4.0 & $0.7 \%$ & 7.0 & $0.6 \%$ & 10.0 & $0.2 \%$ & 13.0 & $0.1 \%$ \\
\hline 1.0 & $2.7 \%$ & 4.1 & $0.7 \%$ & 7.1 & $0.6 \%$ & 10.1 & $0.2 \%$ & 13.1 & $0.1 \%$ \\
\hline 1.1 & $2.4 \%$ & 4.2 & $0.7 \%$ & 7.2 & $0.5 \%$ & 10.2 & $0.2 \%$ & 13.2 & $0.2 \%$ \\
\hline 1.2 & $2.0 \%$ & 4.3 & $0.7 \%$ & 7.3 & $0.5 \%$ & 10.3 & $0.1 \%$ & 13.3 & $0.2 \%$ \\
\hline 1.3 & $1.7 \%$ & 4.4 & $0.8 \%$ & 7.4 & $0.5 \%$ & 10.4 & $0.1 \%$ & 13.4 & $0.2 \%$ \\
\hline 1.4 & $1.4 \%$ & 4.5 & $0.8 \%$ & 7.5 & $0.5 \%$ & 10.5 & $0.1 \%$ & 13.5 & $0.2 \%$ \\
\hline 1.5 & $1.2 \%$ & 4.6 & $0.8 \%$ & 7.6 & $0.5 \%$ & 10.6 & $0.1 \%$ & 13.6 & $0.2 \%$ \\
\hline 1.6 & $1.0 \%$ & 4.7 & $0.8 \%$ & 7.7 & $0.5 \%$ & 10.7 & $0.1 \%$ & 13.7 & $0.2 \%$ \\
\hline 1.7 & $0.8 \%$ & 4.8 & $0.8 \%$ & 7.8 & $0.5 \%$ & 10.8 & $0.1 \%$ & 13.8 & $0.2 \%$ \\
\hline 1.8 & $0.6 \%$ & 4.9 & $0.8 \%$ & 7.9 & $0.5 \%$ & 10.9 & $0.1 \%$ & 13.9 & $0.2 \%$ \\
\hline 1.9 & $0.5 \%$ & 5.0 & $0.8 \%$ & 8.0 & $0.4 \%$ & 11.0 & $0.1 \%$ & 14.0 & $0.2 \%$ \\
\hline 2.0 & $0.3 \%$ & 5.1 & $0.7 \%$ & 8.1 & $0.4 \%$ & 11.1 & $0.1 \%$ & 14.1 & $0.2 \%$ \\
\hline 2.1 & $0.2 \%$ & 5.2 & $0.7 \%$ & 8.2 & $0.4 \%$ & 11.2 & $0.0 \%$ & 14.2 & $0.2 \%$ \\
\hline 2.2 & $0.1 \%$ & 5.3 & $0.7 \%$ & 8.3 & $0.4 \%$ & 11.3 & $0.0 \%$ & 14.3 & $0.2 \%$ \\
\hline 2.3 & $0.0 \%$ & 5.4 & $0.7 \%$ & 8.4 & $0.4 \%$ & 11.4 & $0.0 \%$ & 14.4 & $0.2 \%$ \\
\hline
\end{tabular}




\begin{tabular}{llllllllll}
\hline $\mathbf{2 . 4}$ & $0.1 \%$ & 5.5 & $0.7 \%$ & 8.5 & $0.4 \%$ & 11.5 & $0.0 \%$ & 14.5 & $0.3 \%$ \\
\hline $\mathbf{2 . 5}$ & $0.2 \%$ & 5.6 & $0.7 \%$ & 8.6 & $0.4 \%$ & 11.6 & $0.0 \%$ & 14.6 & $0.3 \%$ \\
\hline $\mathbf{2 . 6}$ & $0.2 \%$ & 5.7 & $0.7 \%$ & 8.7 & $0.3 \%$ & 11.7 & $0.0 \%$ & 14.7 & $0.3 \%$ \\
\hline $\mathbf{2 . 7}$ & $0.3 \%$ & 5.8 & $0.7 \%$ & 8.8 & $0.3 \%$ & 11.8 & $0.0 \%$ & 14.8 & $0.3 \%$ \\
\hline $\mathbf{2 . 8}$ & $0.4 \%$ & 5.9 & $0.7 \%$ & 8.9 & $0.3 \%$ & 11.9 & $0.0 \%$ & 14.9 & $0.3 \%$ \\
\hline $\mathbf{2 . 9}$ & $0.4 \%$ & 6.0 & $0.7 \%$ & 9.0 & $0.3 \%$ & 12.0 & $0.0 \%$ & 15.0 & $0.3 \%$ \\
\hline $\mathbf{3 . 0}$ & $0.5 \%$ & & & & & & & &
\end{tabular}

\section{CONCLUSION}

The mass density calculation method of saturated steam with PLC applications using a linear regression approach can be used as an alternative flow computer that is widely used today with the best accuracy of about $0.2 \%$ in this study. The saturated vapor mass density calculation method which is divided into 5 ranges of values has a better error value which is an average of $0.2 \%$ compared to those not divided into a range of values that have an average error of $0.9 \%$. The use of PLC is good enough to do computational flow according to the formula programmed into PLC memory..

\section{REFERENCES}

[1] Hotanto, TR; Kartika, LGS; Sugiartha, IGRA 2015. Fuel Sales Forecasting System with Simple WebBased Linear Regression Method (Case Application of Gas Station 54,801.38). Journal of JOSINFO: Online Journal of Information Systems, Vol 1. No. 1. thing. - .

[2] Intan Diantari, PN; Kartika, NLA; Dewi, NAN 2015. Vehicle Procurement Forecasting System at Main Motor Award with Linear Regression Method. Journal of JOSINFO: Online Journal of Information Systems, Vol. 1. thing.--

[3] Lawendatu, JR; Kekenusa, JS; Hatidja, D. 2014. Multiple Linear Regression To Analyze the Income of Pala Farmers. Journal of De Cartesian, Vol 3. No 1. 66-72.

[4] Syafruddin, M .; Lukmanul, H .; Dikpride, D. 2014. Linear Regression Methods for Prediction of LongTerm Electric Energy Needs (Case Study of Lampung Province). Journal of JITET: Journal of Informatics and Applied Electrical Engineering, Vol. 1, No. 2. Hal.-.

[5] pirax Sarco. (2017, October 25). Retrieved 0ctober 25, 2017, from Spirax Sarco. 